\title{
Chapter
}

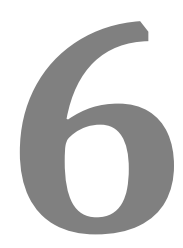

\section{NOVEL DRUG DELIVERY SYSTEMS TO COMBAT ANTIMICROBIAL RESISTANCE}

Pranothi Mulinti, Raquib Hasan, and Amanda Brooks*

Department of Pharmaceutical Sciences, North Dakota State University, Fargo, ND, 58105 


\section{Contents}

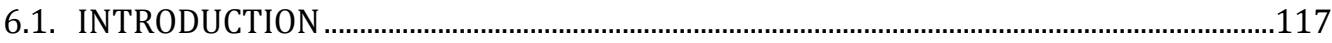

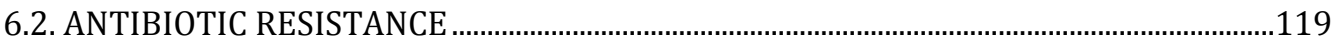

6.2.1. Mechanism of resistance ...................................................................................19

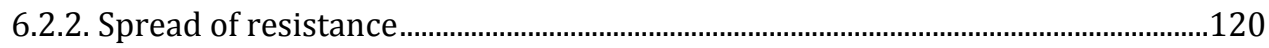

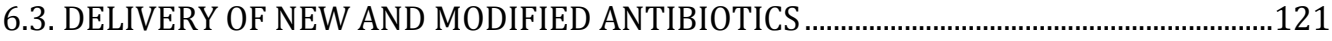

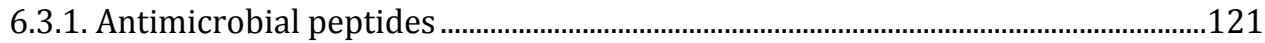

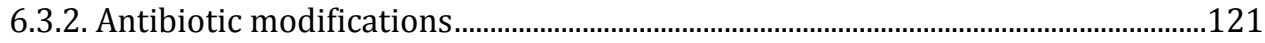

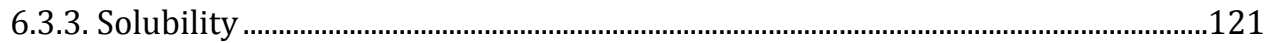

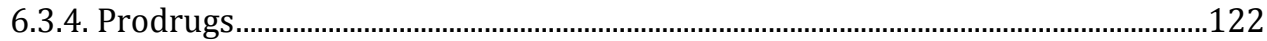

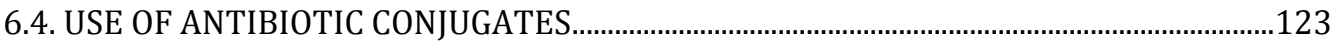

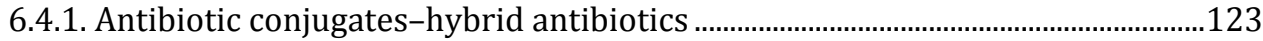

6.4.2. Nanoparticle/antibiotic conjugates........................................................................124

6.4.3. Antibiotic adjuvant combinations .........................................................................126

6.5. TRADITIONAL AND EMERGING APPROACHES TO COMBAT RESISTANCE-

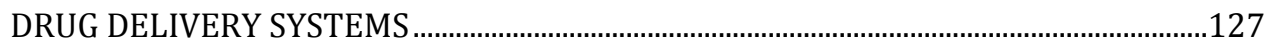

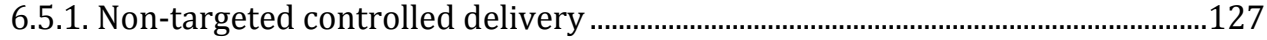

6.5.2. Infection responsive drug delivery systems .............................................................128

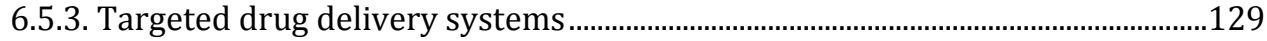

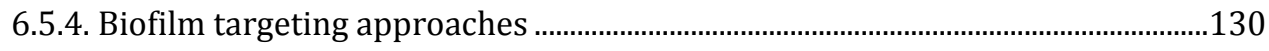

6.6. OTHER EMERGING APPROACHES AND CARRIERS ……....................................................132

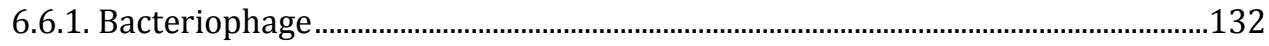

6.6.2. Antibiotic drug discovery and development..............................................................132

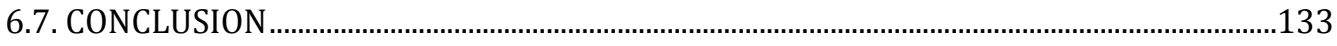

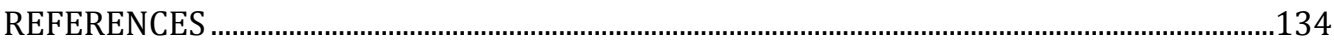




\subsection{INTRODUCTION}

Although not the first antibiotic discovered, the introduction and development of penicillin to widespread use, changed the face of healthcare and effectively launched an arms race with pathogenic bacteria [1]. Despite the benefits of antibiotics, the evolution and development of antibiotic resistant bacteria have been noted within 20 years of their introduction into therapeutic practice (Table 1). Gram-negative bacterial resistance is of particular concern as there are very few available treatment options following the emergence of resistance.

Table 1: Emergence of resistance against some antibiotics after the start of use (modified from [2])

\begin{tabular}{c|c|c|c}
\hline Antibiotic & $\begin{array}{c}\text { Year of } \\
\text { introduction as } \\
\text { therapeutic }\end{array}$ & $\begin{array}{c}\text { First report of } \\
\text { resistance }\end{array}$ & $\begin{array}{c}\text { Time (in years) taken } \\
\text { for resistance to occur }\end{array}$ \\
\hline Penicillin & $\begin{array}{c}1943 \\
\text { discovered in } \\
1928)\end{array}$ & $\begin{array}{c}1940 \\
\text { before starting } \\
\text { mass use) }\end{array}$ & $\begin{array}{c}\text { 12 years after discovery } \\
\text { but 3 years before the } \\
\text { start of mass use [1] }\end{array}$ \\
\hline Tetracycline & 1950 & 1959 & 9 \\
\hline Erythromycin & 1953 & 1968 & 15 \\
\hline Methicillin & 1960 & 1962 & 12 \\
\hline Gentamicin & 1967 & 1979 & 13 \\
\hline Vancomycin & 1972 & 1988 & 2 \\
\hline Imipenem & 1985 & 1998 & 0 \\
\hline Ceftazidime & 1985 & 1987 & 1 \\
\hline Levofloxacin & 1996 & 1996 & $13]$ \\
\hline Linezolid & 2000 & 2001 & 16 \\
\hline Daptomycin & 2003 & $2005(\mathrm{rare})$ & 2011 \\
\hline Ceftaroline & 2010 & &
\end{tabular}

The threat of antibiotic resistance has reached alarming proportion with more than 2 million individuals being admitted to the hospital each year in United States, culminating in 23,000 deaths and an overall financial burden reaching 35 billion USD [4]. Unless drastic measures are taken soon, grim predictions estimate the annual worldwide death toll may increase to 10 million per year by 2050 [5]. To exacerbate the situation, the drive towards the development of newer antibiotics has slowed down [6]. The Food and Drug Administration (FDA) approved 49 new antibiotics during 1981-2000 period, out of which 24 
were later withdrawn or discontinued, compared to only 16 between 20012015 [7]. Worldwide, during the period from 2000-2015 30 new antibiotics were marketed along with two new $\beta$-lactam $/ \beta$-lactamase inhibitor combinations (BBLIC) [6] (Figure 1). Note that $\beta$-lactamase will breakdown penicillin and lead to resistance. The drop in approvals may indicate, either a changing, stricter FDA approval process, fewer submissions, or a lack of pharmaceutical industry support for development. Out of the new drugs approved, five were totally new classes of antibiotics, but none were targeted against gram negative bacteria [6].

The overuse and misuse of antibiotics in both healthcare and agriculture, including unnecessary or incorrect prescriptions, poor patient compliance, rapid bacterial evolution in response to bactericidal selective pressure, have led to rising resistance. Improved infectious pathogen diagnostics as well as a relatively dried up discovery and approval pipeline have led to the current crisis [8-10]. In this context, distinctly new approaches to delay or circumvent the emergence of resistance while we await newer antibiotics and novel ways to treat bacterial infections are needed. This chapter will specifically focus on the emerging approaches to combat antibiotic resistance, specifically the development and use of novel drug delivery systems to fight bacterial resistance.

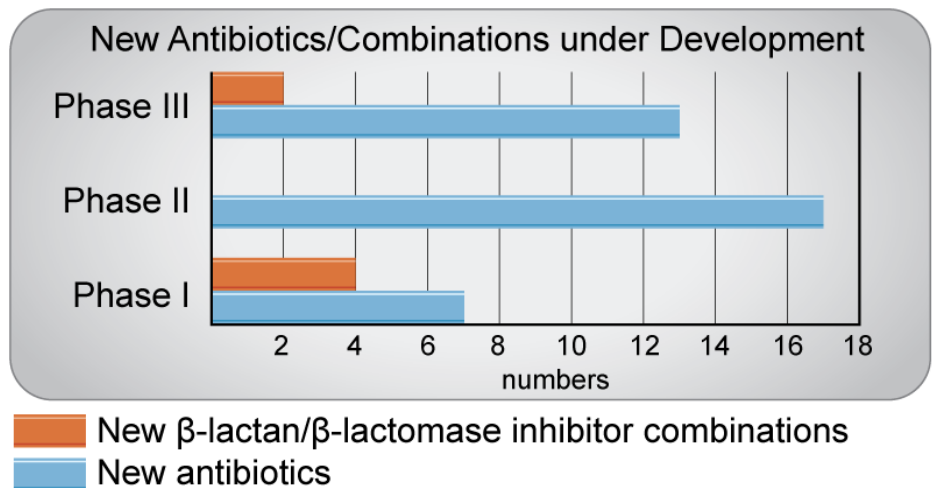

Figure 1. New antibiotic and BBLIC under development as of 2015 


\subsection{ANTIBIOTIC RESISTANCE}

\subsubsection{Mechanism of resistance}

Although the mechanisms of resistance will not be thoroughly covered as several authors have recently published multiple good reviews [1,2,11,12], a general overview of the topic is provided here to provide context for the intelligent design of novel drug delivery systems to fight resistance. Bacteria can develop antibiotic resistance through a variety of diverse mechanisms. Of these, innate genetic mutations can be seen as a prime cause due to their short generation time and rapid evolution [13]. Mutations that confer resistance provide a fitness advantage and lead to elimination of "less fit", susceptible strains $[13,14]$. Resistance via mutation can be considered one form of adaptive resistance. Additionally, adaptive resistance may also be due to environmentally triggered genetic changes such as nutrient conditions, growth state, subtherapeutic concentrations of antibiotics itself [15], etc. Alternatively, bacteria can also gain resistance from other resistant strains via horizontal gene transfer (HGT) (plasmid, transposons, integrons, etc.) during conjugation-"acquired resistance" $[15,16]$.

Regardless of the mechanism, of great concern is the resistance acquired in the presence of an antimicrobial agent. Resistant bacteria can then spread and proliferate under antibiotic selective pressure [17]. Several different genes and pathways may be targeted during resistance acquisition. Specifically, bacteria can modify antibiotics by altering (i) genes involved in the synthesis and positioning of the antibiotic target and the access pathways of the antibiotic into the target. These mutations cause modifications on the cell surface by changing the permeability of the plasma membrane, which leads to reduced entry of the drug into the cell, thereby reducing the interactions between the bacteria and the antibiotic and leading to reduction in the production of entry channels like porins further restricting the entry of the drug inside the cell. (ii) Target protecting genes such as those involved in modification or inactivation of enzymes. Bacteria can synthesize antibiotic modifying enzymes that selectively target and degrade the activity of antibiotics by either hydrolysis, as in the case of $\beta$-lactamases, group transfer for aminoglycosides, or redox mechanisms. (iii) Genes involved in changing the cell permeability and modifying the efflux pumps. These pumps are naturally occurring in the bacteria to prevent the accumulation of toxic compounds inside the cell. Upon extended exposure to antibiotics, bacteria can modify these pumps to efflux antibiotics outside the cell. Thus, even the antibiotic reaches inside the cell by escaping other resistant mechanisms, it can still be actively pumped out $[15,18,19]$.

In addition to both direct adaptive and acquired resistance, biofilm formation, where planktonic bacteria irreversibly attach to a surface and are covered by a thick exopolysaccharide (EPS) biopolymer layer made of polysaccharides, 
proteins and DNA, represents another important mechanism for the development of resistance. As the biofilm grows in thickness and matures, the bacterial members show maximum resistance to antibiotics [20]. Importantly, while bacteria persist in a biofilm, chronic infection ensues. Alternatively, as soon as the bacteria are redispersed from the biofilm, antibiotic sensitivity is restored. The rapid reversal of resistance from the redispersion of biofilm bacteria suggests an adaptive resistance more than a genetic alteration, although there are elements of genetic alteration as well [21]. Furthermore, several reports have also demonstrated increased mutation frequency in biofilm forming bacteria, which in turn facilitates effective HGT by conjugation and transformation $[20,22]$.

\subsubsection{Spread of resistance}

Genetically, resistance can spread from one bacteria to another by both "vertical," and "horizontal" methods. During a vertical transfer, parental bacteria pass in antibiotic resistance genes to their progeny. Alternatively, during HGT, bacteria share or exchange genetic material during mating. Importantly, HGT can even occur between different bacterial species. Additionally, bacteriophages may act as vectors for passing resistance traits between bacteria [23]. Apart from vectors, bacteria themselves can also acquire naked DNA from their environment. Furthermore, bacteria move themselves from place to place and spread resistance in the environment.

While the globalization of bacterial species is increasingly common and an important factor for controlling the spread of antibacterial resistance, avoidance of bacterial spread is almost impossible in any environmental setting. Thus, the proper use of antibiotics is essential to prevent further bacterial resistance from emerging. The Centers for Disease Control recently reported in their 2017 report on Antibiotic Use in the United States, that $30 \%$ of antibiotic prescriptions in both the hospital and outpatient settings are not only unnecessary but are also inappropriately prescribed with providers favoring broad-spectrum and last line antibiotics over more targeted, first-line approaches [24]. About $90 \%$ of the antibiotics prescribed are given by general practitioners and most inappropriate use in outpatient settings is prescribing antibiotics for viral infections and respiratory infections [25]. These prescribing patterns also lead to increased risk of side effects [1] and elevated economic burden on the already taxed healthcare system. Nevertheless, we do not find ourselves of the precipice of a return to the pre-antibiotic era of medicine strictly due to prescribers; patients also bear a significant responsibility due to poor compliance, and lack of knowledge that contributes to self-medication, a common practice in developing countries [26]. Irrespective of the type of mechanism of resistance and the spread of resistance, combatting it requires multiple levels of intervention, including new and modified antibiotics, antibiotic conjugates, and cutting edge drug delivery systems, to address critically this rising global problem. 


\subsection{DELIVERY OF NEW AND MODIFIED ANTIBIOTICS}

\subsubsection{Antimicrobial peptides}

Antimicrobial peptides (AMPs) are a diverse group of molecules that (1) show potent immune modulatory activity and antimicrobial function and (2) are produced by many tissues and cell types in a variety of invertebrate, plant and animal species [27]. AMPs generally consists of 10-50 amino-acid residues, which possess certain common features like amphipathic structure, net positive charge and rapid binding to biological membranes [28]. They are divided into different subcategories based on their structure and amino acid composition and are known to provide broad activity against bacteria, parasites, fungi and viruses. Although their mode of action is still not fully understood, reviews show that cytoplasmic membranes and intracellular molecules are the major targets for AMPs, with the mechanism of action being based on a combination of (1) neutralization of lipopolysaccharide, (2) inhibition of cell wall synthesis, (3) alteration of the membrane potential, and (4) inhibition of proteins involved in cell division [28]. Although resistance to AMPs has been reported, it is very difficult to acquire due to their rapid killing action. AMPs have been shown to be particularly effective delivered in combination with current antibiotics against multi drug resistant (MDR) bacteria [29].

\subsubsection{Antibiotic modifications}

Altering the antibiotic formulation including (1) targeting them to the site of infection, (2) enhancing selective uptake by bacteria, (3) increasing penetration inside cells to attack infiltrated bacteria, or (4) increasing the local concentration of antibiotic can all be strategies to control and improve antibiotic therapy and thwart the resistance process. Although these strategies have been recognized to improve delivery and efficacy, several methods are available to provide such modifications.

\subsubsection{Solubility}

Since the introduction of the antibiotic to modern medical practice, several older antibiotics have been abandoned due to toxicity; however, several modifications and new delivery systems may allow for their reintroduction with minimal toxicity. In the early 1980s, the use of polymyxin E (colistin) was limited due to reports of renal toxicity. However, revival of this drug to treat several resistant gram-negative bacterial infections, in less toxic form, may prove to be useful [30]. Hence, colistin sodium found to be less toxic than colistin sulfate. Modifying the solubility of the drug will change its bioavailability thereby altering its toxicity. In the case of poorly soluble antibiotics, modification to a salt form of the drug with an organic counter ion will improve its solubility and bioavailability. Additionally, the solubility of an 
antibiotic can be controlled based on $\mathrm{pH}$ [31]. However, as a hydrochloride salt form, it shows slow dissolution in stomach at acidic $\mathrm{pH}$ due to common ion effect [31]. Ciprofloxacin shows low solubility at neutral $\mathrm{pH}$, leading to low bioavailability. Alternatively, the addition of carboxylic acid salts, oxalate, tartarate, benzoate, malonate, and citrate, to nofloxacin or ciprofloxacin showed higher solubility and faster dissolution at $\mathrm{pH} 6.4$ in pure water and at 6.8 in phosphate buffered saline (PBS), though the opposite effect was observed at an acidic pH of 1.2 [31]. Changing the solubility of the parent compound via addition of an organic counter ion can also stabilize the parent compound, as demonstrated by the improved stability of norfloxacin, which is transformed into dihydrate at similar conditions [32]. Antibiotics with improved stability or enhanced delivery at specific $\mathrm{pH}$ have been exploited in a variety of new drug delivery systems [33,34].

\subsubsection{Prodrugs}

Not only can altering the $\mathrm{pH}$ and solubility of a parent compound change its bioavailability but it can also hasten the pharmacokinetics of drug release, necessitating further drug modifications. A polymeric prodrug, which must be metabolized to release the active drug (Figure 2), provides additional control over drug solubility, targeted drug delivery, and antibacterial efficacy and can be an attractive alternative to avoid the rapid development of antibiotic resistance in response to an otherwise ubiquitous antibiotic environment [35].

A polymeric ciprofloxacin prodrug, ciprofloxacin-(phenol)methacrylate (CPM) and PEGMA 950 (0950) conjugate (poly(0950-co-CPM)), made via Reversible Addition-Fragmentation chain Transfer (RAFT) polymerization achieved high drug loading, about $16.5 \% \mathrm{wt}$ and showed good in vitro antibacterial activity [35]. CPM also showed very good in vivo activity in a francisella tularensis subsp. novicida (F.t. novicida) mouse infection model with $75 \%$ survival rate, when administered via endotracheal aerosolization, compared $0 \%$ in case of when free ciprofloxacin and slow releasing ciprofloxacin from alkyllic ester conjugate was administered. CPM, with its fast, controlled and sustained delivery kinetics and ability to attack bacteria intracellularly in alveola proved to be lifesaving in the experiment against deadly F.t. novicida infection [36]. 


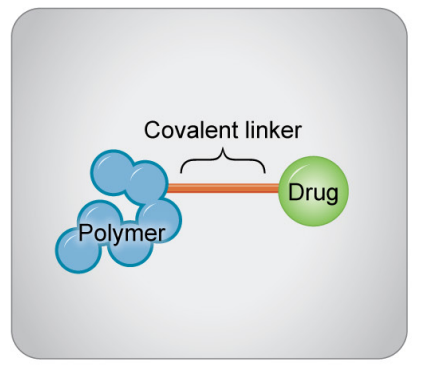

Polymeric Prodrug

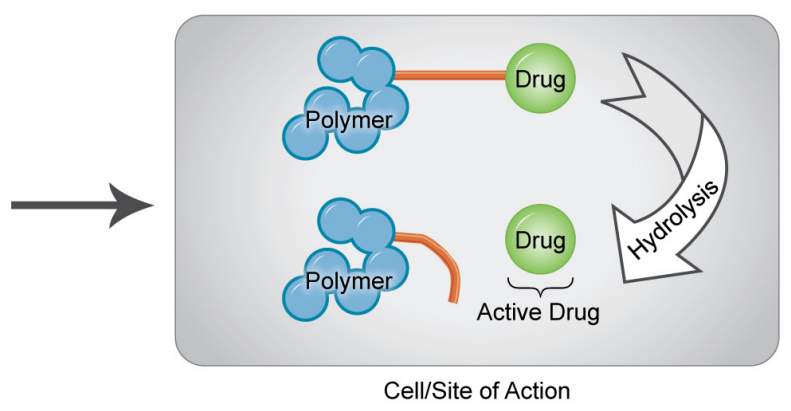

Figure 2. Polymeric prodrugs can be cleaved through hydrolysis to provide active drug. Delivery of a prodrug can protect the active drug allowing longer circulation and extended activity.

\subsection{USE OF ANTIBIOTIC CONJUGATES}

\subsubsection{Antibiotic conjugates-hybrid antibiotics}

Although the rapid discovery of new antibiotics has slowed to a crawl in the face of rising resistance, a unique way to counter resistance is being explored; conjugating antibiotics with complementary mechanisms to make a hybrid antibiotic is proving to be a value and evolving field of discovery (Figure 3 ). This approach can be especially effective in combatting MDR bacteria by increasing an antibiotic's (1) penetration, (2) bioavailability, and (3) spectrum of activity while concurrently reducing its toxicity and risk of resistance through the use of an integrated combination therapy that works synergistically [37]. Similar to the cleavable linkers of prodrugs, two antibiotics with complimentary mechanisms of action can be linked to create a hybrid. Alternatively, a non-cleavable bond may also be used when the hybrid is meant to act as a dual-functioning drug.

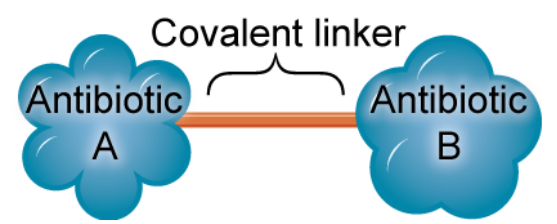

Figure 3. Antibiotic Hybrids can be created by using a cleavable or non-cleavable covalent bond to link two drugs with complimentary mechanisms of action together 
Hybrid oxazolidinone-quinolone antibiotics showed improved activity against susceptible as well as resistant gram positive and negative bacteria. While oxazolidinones have limited permeability into gram-positive bacteria, which reduces their ability to reach their RNA target, hybridizing it to a quinolone, which targets DNA topoisomerase/gyrase and has good permeability helps oxazolidinones reach its target, thus improving its efficacy. Oxazolidinones, hybridized with ciprofloxacin, ofloxacin, and levofloxacin respectively via a piperazine group showed high potency against linezolid-susceptible Staphylcoccus aureus and E. faecium, gram positive bacteria, with minimum inhibitory concentration (MIC) $\leq 1 \mu \mathrm{g} \mathrm{mL}^{-1}$. The hybrids were also active against linezolid and quinolone resistant strains, showing 32-128 fold more activity [38].

While the oxazolidinone-quinolone hybrid provides broad applicability for both gram-positive and gram-negative bacteria, other hybrids are designed to target only specific bacteria, protecting the delicate microbiota balance in body. MBX-500, a hybrid of an anilinuracil, a DNA polymerase inhibitor, and a fluoroquinolone is a unique in that it has selective efficacy against a highly virulent strain of $C$. difficile but leaves most other gram-negative and grampositive bacteria unaffected. This targeting ability could render it an antibacterial agent less susceptible to bacterial resistance [39]. Alternatively, other hybrids focus on multivalency, which may improve their affinity towards targeted bacteria. Multivalency can be defined as multiple interaction between ligand and its target, leading to a strong affinity $[40,41]$. Vancomycin resistant Enterococci (VRE) is a particular threat. Vancomycin and nisin are two different classes of antibiotics, both attacking lipid II of bacterial cell wall, but the target affinity is for two different components of lipid II: for vancomycin, it is tripeptide part (Lys-D-Ala-D-Ala), while for nisin, its $N$-terminal fragment (residues 1-12) binds to the pyrophosphate part. A hybrid of these two may end up increasing the multivalent affinity toward two different components of lipid II, rendering it a potent drug. The vancomycin-nisin (1-12) hybrid was made through click chemistry. Several different linkers were evaluated, and the hybrid linked by three polyethylene glycol (PEG) units (linker length $12.2 \mathrm{~A}$ ) showed the most activity. The hybrid was 40 times more active against VRE when compared to the activity of the individual components. It also showed good activity against gram-negative Klebsiella pneumoniae, against which vancomycin alone is typically ineffective [41].

\subsubsection{Nanoparticle/antibiotic conjugates}

Another strategy to restore antibacterial activity against resistant bacteria is to conjugate the antibiotic with a nanoparticle. Vancomycin resistant $S$. aureus (VRSA) has been shown to be 6 times more susceptible to vancomycin conjugated with gold nanoparticles (VGNP) (minimum inhibitory concentration, MIC, is $6 \mu \mathrm{g} \mathrm{mL}^{-1}$ ) when compared to unconjugated vancomycin. VGNP is hypothesized to have unspecific binding to transpeptidases in cell wall 
of VRSA instead of specific binding to the terminal peptides (d-Lac) of VRSA. Importantly, bacteria with mutated d-Lac are resistant to vancomycin. VGNP also showed activity against E. coli. Generally, vancomycin does not show therapeutic activity against gram-negative bacteria due to hindrance of the outer membrane. Despite its larger size, it is hypothesized that VGNP crosses the membrane and destabilizes the lipopolysaccharide membrane, thus allowing vancomycin to bind to the mucopeptide region, causing cell wall lysis. Although this occurs at relatively high MIC $\left(40 \mu \mathrm{g} \mathrm{mL}^{-1}\right)$, it is lower than that for unconjugated vancomycin $\left(175 \mu \mathrm{g} \mathrm{mL}^{-1}\right)$ [42]. Even though this strategy, in its current form, may not be clinically relevant to treat gram-negative infection, it provides a new route for possible exploration.

While gold nanoparticles may themselves demonstrate antimicrobial activity [43], polyacrylate nanoparticles, which have no known independent antibiotic activity, can be bound to penicillin and penicillin derivatives to restore their activity in methicillin resistant $S$. aureus (MRSA). Penicillin G linked with acrylate via a hydrolysis susceptible covalent bond enhanced activity against MRSA as well as methicillin susceptible $S$. aureus (MSSA) when compared to other formulations. It was 8 times more active against MRSA compared to free penicillin G, having an MIC of $2 \mu \mathrm{g} \mathrm{mL}^{-1}$. Alternatively, another formulation using an ester linkage between the acrylate particle and penicillin $\mathrm{G}$ was not as potent. Non-covalently conjugated, encapsulated drug did not show any notable activity. Covalently bound nanoparticle conjugates such as these have been hypothesized to help the drug to reach the bacterial cell while protecting it from bacterial modifying and inactivating enzymes such as $\beta$-lactamase. The release of drug at the site of action may involve hydrolysis and/or esterase activity as well as interaction of nanoparticle with bacterial cell membrane [44].
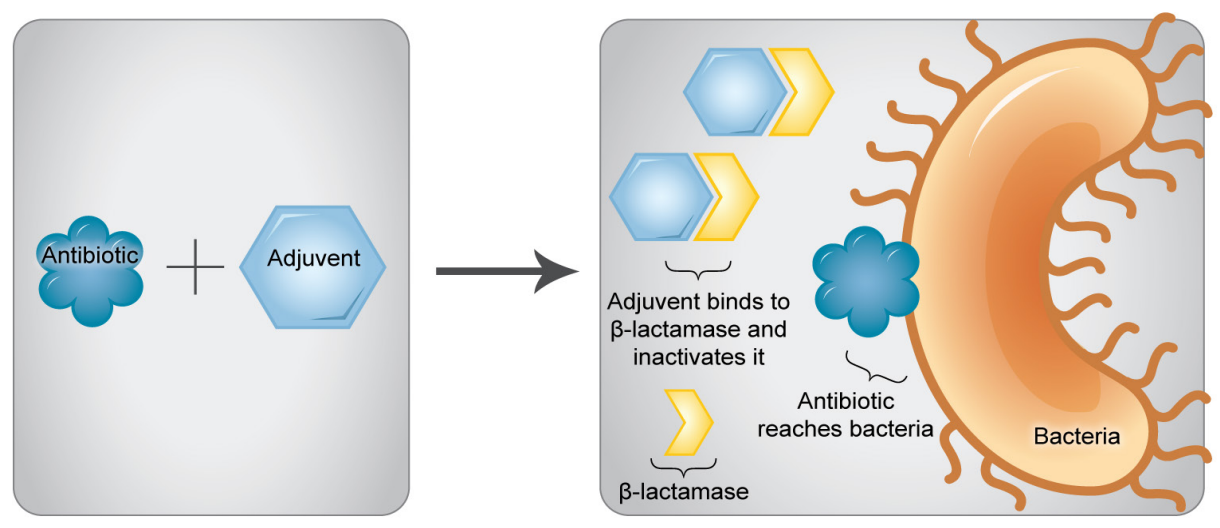

Figure 3. When an antibiotic is delivered with an adjuvant, the adjuvant can bind the $\beta$-lactamase, allowing the antibiotic to reach its bacterial target intact and active 


\subsubsection{Antibiotic adjuvant combinations}

While nanoparticle conjugates can be seen as carriers to enhance the activity of their antibiotic partner, adjuvants may also play a critical role in the prevention of bacterial resistance as well as the restoration of antibacterial activity of antibiotics against resistant bacteria. Based on their mechanism, adjuvants can be divided into two main classes. Type I adjuvants block bacterial resistance mechanisms by inhibiting antibiotic degrading enzymes, inactivating efflux pump, etc. Conversely, Type II adjuvants work on the host and potentiate the antibiotic's action. Only type I adjuvants, which work against $\beta$-lactamase and extended-spectrum $\beta$-Lactamases (ESBLs), are clinically used. $\beta$-lactamase activity plays a crucial role in bacterial resistance against antibiotics containing $\beta$-lactam ring [45]. $\beta$-lactamase breaks the $\beta$ lactam ring open, rendering the antibiotic incapable of its activity. Thus, $\beta$ lactamase related resistance is wide spread, with more than $1400 \beta$-lactamases identified [46]. $\beta$-lactamase inhibitors (BLI) and ESBLs can render penicillins, carbapenems, monobactams and cephalosporins susceptible to bacterialdriven inactivation and resistance. These enzymes can also be secreted from bacterial cells, hydrolyzing the drug even before it reaches the bacteria [47]. In an effort to combat antibiotic inactivating enzymes such as $\beta$-lactamase, adjuvants, which first appeared in the 1970s and have little to no independent antibacterial activity, can be delivered with the antibiotic to inhibit and counter enzymes like $\beta$-lactamases by irreversibly binding to $\beta$-lactamases, protecting the drugs from breaking down [46] (Figure 4). Clavulanic acid, a $\beta$ lactamases inhibitor (BLI), was the first adjuvant used in clinical practice [48]. $\beta$-lactam $/ \beta$-lactamases inhibitor combinations (BBLIC) can be powerful weapons against antimicrobial resistance.

Studies showed a decreased rate of resistance emergence when BBLICs were used when compared to antibiotic only. Additionally, in Taiwan, $K$ pneumoniae bacteraemia treated with piperacillin-tazobactam showed decreased risk of ESBL positive isolates compared to previous exposure to oxyimino- $\beta$-lactam. Furthermore, four Australian intensive care units reported lower rates of MRSA and Pseudomonas aeruginosa infection when BBLICs were used in contrast to cefepime treatment. Piperacillin-tazobactam therapy, as opposed to carbapenems, produced a decline in carbapenem-resistant Enterobacteriaceae, in Illinois. In the UK, a change in prescribing habits have trended towards use of BBLICs and away from third-generation cephalosporins, which have led to a decrease in cephalosporin resistance in Enterobacteriaceae isolated from bloodstream infections. The therapy did not produce any increase in piperacillin-tazobactam resistance [49]. In India, Cefepime-tazobactam and ceftriaxone-sulbactam combinations are now available in the market; both showing good in vitro efficacy against in different clinical isolates [49]. Ceftolozane/tazobactam and ceftazidime/avibactam combinations got FDA approval in 2014 and 2015, respectively [7]. Avibactam is a unique and novel BLI. It is a covalent, reversible, non- $\beta$-lactam $\beta$-lactamase 
inhibitor. It is the most potent BLI to date with a $1-5: 1$ ratio of avibactam: $\beta$ lactamase needed for inhibition, compared to $>50: 1$, BLI: $\beta$-lactamase molecule, for tazabactam and clavulanate [46]. Moreover, ceftolozane/tazobactam have performed well against ESBL producing Enterobacteriaceae and $K$. pneumoniae infections in clinical studies [46]. Clavulanic acid, tazobactam, sulbactam and avibactam are adjuvants in use. Aspergillomarasmine A (AMA) is a potent metallo- $\beta$-lactamase (MBL) inhibitor that recently showed promise in irreversibly binding and inactivating MBL both in vitro and in vivo. When the mice were treated with a meropenem and AMA combination, the survival rate of mice infected with a lethal NDM-1 positive K. pneumoniae load was more than $95 \%$ with a single dose compared to $0 \%$ in case of meropenem alone [50]. The combination of imipenem/relebactam significantly lowered the MIC and showed synergistic killing of resistant strains of $P$. aeruginosa and $K$. pneumoniae in several in vitro studies [46].

\subsection{TRADITIONAL AND EMERGING APPROACHES TO COMBAT RESISTANCE-DRUG DELIVERY SYSTEMS}

\subsubsection{Non-targeted controlled delivery}

Alterations in the drug can also change the pharmacokinetics of drug release, which may prove an effective alternative to delay the emergence of antibiotic resistance. Controlled extended release formulations have long been used to treat disease conditions; however, for infectious conditions extended antibiotic release can inadvertently promote the development of bacterial resistance (e.g., antibiotic loaded bone cement). Controlling the pharmacokinetics of local extended release formulations via the physical properties (i.e., solubility, pKa, etc.) of the drug may mitigate this risk while still providing the advantages of local controlled release. Making a hydrophilic antibiotic less soluble can be a viable strategy to extend its release [51]. This phenomenon has been observed with local vancomycin release from a bone graft void filler [52]. The clinical form of vancomycin, vancomycin $\mathrm{HCl}$, was exhausted from its bone graft depot within 2-3 weeks; whereas, release of the desalted form of vancomycin was extended to 7 weeks (unpublished data). Gentamicin sodium bis(2ethylhexyl)sulfosuccinate (AOT), a less soluble form of gentamicin, in PLGA nanoparticle showed release up to 70 days [52]. Other fatty acid derivatives of gentamicin such as gentamicin sodium dodecyl sulfate, gentamicin laurate and gentamicin palmitate also showed slower release from vascular prostheses [53]. Collagen fleece containing readily soluble gentamicin sulfate and gentamicin crobefate, a hydrophobic modification of gentamicin, showed slow 
and sustained release for up to 12 days with initial high concentration due to the hydrophilic part and sustained release due to the hydrophobic form [54].

Of particular concern for extended release formulations in the development of antibiotic resistance is the consistent presence of antibiotic, which can drive bacteria to infiltrate host cells. Thus, treatment of intracellular bacteria must be considered with formulations designed specifically for this challenging case. Phagocytic cells easily take up liposomes, providing a mechanism for intracellular antibiotic drug delivery. Intracellular lethal bacteria, F. tularensis, can be treated via liposomal delivery of ciprofloxacin. In a F. tularensis mouse infection model, this liposomal ciprofloxacin preparation showed significantly better survival than free ciprofloxacin [55]. Another study with liposome encapsulated ciprofloxacin demonstrated enhanced phagocytic functions of macrophage, in turn showing antibacterial activity against $S$. aureus via two mechanisms: activity due to antibiotic and activity due to increased phagocytic function. By addressing intracellular colonization, not only can primary infection be addressed but also secondary, infectious relapses may be minimized [56].

\subsubsection{Infection responsive drug delivery systems}

Drug delivery systems that respond to molecular cues unique to tissues or disease conditions have recently gained widespread scientific interest. Many disease conditions have been characterized by upregulation of specific proteases, which can act as therapeutic targets, disease markers, etc. [57]. Several enzymes were reported to be elevated during infection including a thrombin-like enzyme in S. aureus, myeloperoxidase in Candida, and esterases in uropathogens. These enzymes can be used as biomarkers for timely initiation of treatment and also as therapeutic targets for infection responsive drug release system (Figure 5) [5859]. S. aureus and P. aeruginosa infected wound fluid has been reported to have high thrombin-like activity due to bacterial enzymatic activity [60]; whereas, the release of bacterial phosphatase and phospholipase, which are key virulence factors for certain bacteria, are reported in other studies [61]. Likewise, elevated concentrations of myeloperoxidase released from neutrophils and monocytes have been reported during the inflammatory response to infection [62,63], while macrophages produced cholesterol esterase during infection [64]. Infection with intracellular pathogens like Mycobacterium tuberculosis was also shown to increase glycolysis, including glycolytic enzymes and glucose transporters, while downregulating tricarboxylic acid cycle, suggesting that immune cells prefer aerobic glycolysis during tuberculosis infection [65]. These unique biological cues can be exploited during the develop of novel, infectiontriggered, local drug release systems, which not only increase therapeutic efficacy, but also decrease toxic systemic side effects. Importantly, such drug delivery systems would not necessarily need to be targeted. 

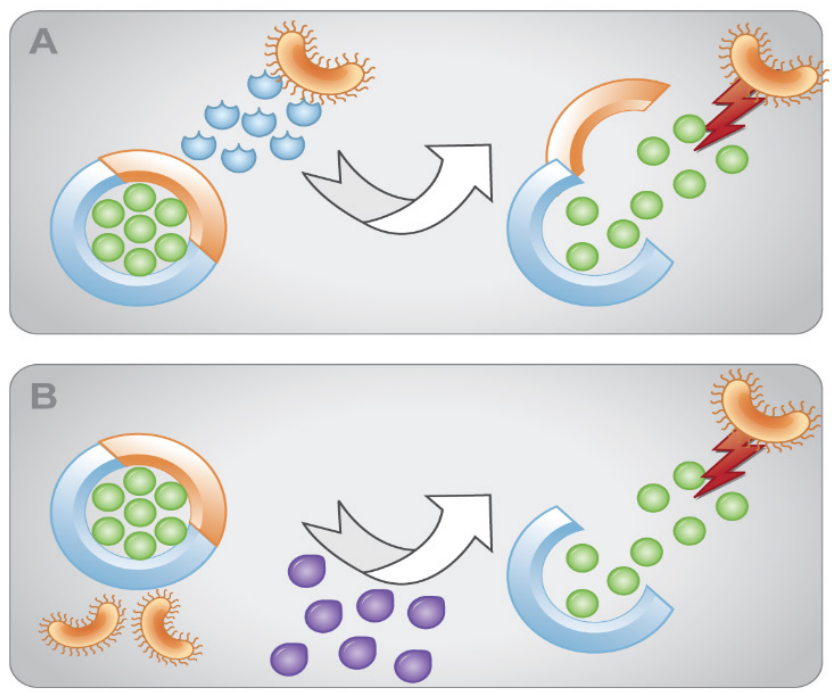

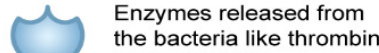
phospholipase

Infection responsive element

Drug molecule

Elevated concentration of molecules like

myeloperoxidase and cholesteral esterase during infection

Drug carrying vehicle

Figure 5. A) Enzymes (e.g., thrombin, phospholipase) released from the bacteria during infection act as a trigger to release antibiotics locally at the site of infection.

B) During infection, the elevated concentration of molecules such as myeloperoxidase and cholesterol esterase acts as a trigger to release the antibiotic from the vehicle.

\subsubsection{Targeted drug delivery systems}

Clostridium difficile is the most common infectious, diarrhea-causing organism that resides in the human colon. Upon initiation of antibiotic therapy, the normal gut flora was disrupted, enabling C. difficile to persist in the gut as the dominant bacteria and induce colitis $[66,67]$. Keeping in mind that virulence if often tied to resistance [68], the emergence of highly virulent strains of this bacteria and a reduced susceptibility to antibiotics has led to the development of MDR strains [69]. Fortunately, recent studies have shown that certain lytic enzymes from bacteria and bacteriophages can act as highly selective and effective antimicrobials [70]. These enzymes can be delivered without a traditional targeted delivery system and yet still remain selective against a wide range of $C$. difficile, preserving the normal gut flora.

Chlamydia trachomatis (CT), which grows in the genital mucosal cells, is the leading cause of sexually transmitted diseases [71]. CT is an intracellular pathogen residing inside the host cell in a protective inclusion body, which has selective interactions with the exocytic pathways [72]. This niche of protective layers limits the accessibility of antibiotics. Recent findings suggest the importance of the transferrin iron pathway as an intracellular drug carrier. Iron containing transferrin is internalized into the endosome of the cell where 
transferrin is gradually acidified to release iron into the cytoplasm [73]. In a similar manner, antibiotics can be loaded into transferrin where it can act as a vehicle to deliver the drug into the cells. Utilizing bacteria's own iron scavengers and transporters is a promising strategy to combat the resistance of CT to a broad variety of antibiotics.

Similar to CT, tuberculosis remains worldwide public health threat affecting $1 \%$ of the total population every year, making it the number one cause of death due to infectious diseases [74]. Additionally, Mycobacterium tuberculosis, is an intracellular pathogen, which can be phagocytosed by alveolar macrophages (AM) where they adapt to the host cell microenvironment to survive for years [75]. One of the prime factors for the survival of $M$. tuberculosis inside the AM is the specific interaction between the pathogen and the host, which occurs through various cell surface receptors including mannose receptor [76]. The virulent $M$. tuberculosis has a unique cell wall mannose-capped lipoarabinomannan, which interacts with the mannose receptor on the macrophages to enter into these cells [77,78]. This transporter pathway can be hijacked in a similar fashion by coating drug carrying vehicles with mannose receptor recognition sequences to target AM intracellular bacteria.

\subsubsection{Biofilm targeting approaches}

No discussion of antibiotic resistance would be complete without considering the unique and challenging case of drug delivery to biofilms. Biofilms are a cluster of microbes grouped together and attached to a surface protected by secreted extra cellular matrix (ECM)/extracellular polymeric substance (EPS). This transition of bacteria from planktonic to sessile occurs due to environmental changes and gene expression changes, which modify the expression of surface molecules, thereby changing the virulence of the bacteria and altering the nutrient utilization of the bacteria [79]. The complex structure of the ECM/EPS and the constant changing behavior of the biofilm architecture makes a biofilm highly tolerant towards antibiotic treatment. At the most rudimentary level, mature biofilms can be disturbed by mechanical means using water sprays and jets along with debridement of surgical site infections [81]. Alternatively, biofilm formation can be targeted at various stages throughout their life cycle [80] (Figure 6). 


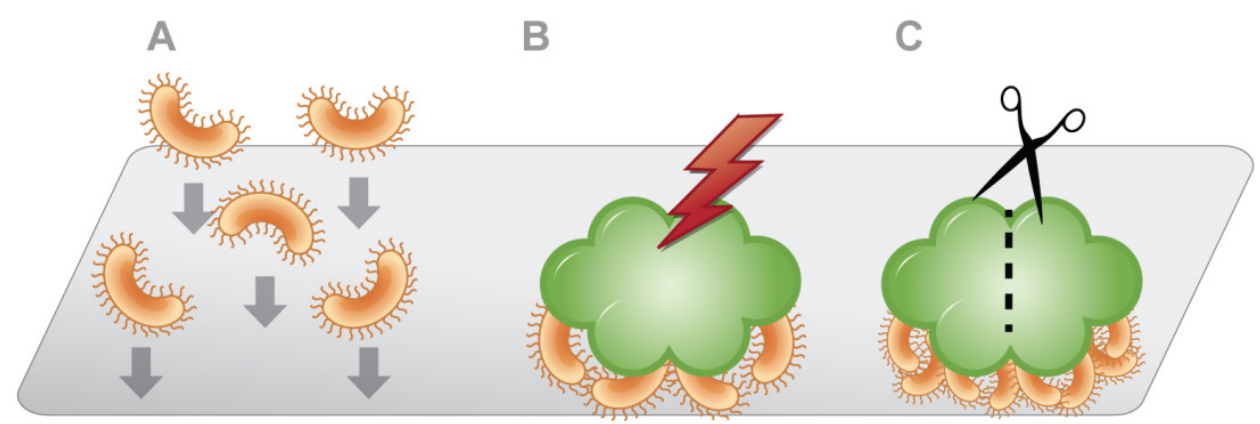

Figure 6. Mechanisms to target biofilms either A) prevent the initial attachment of bacteria to the surface, B) inhibit of production of EPS, or C) surgically debride and physical remove the formed biofilm

During the initial phase of biofilm formation, bacteria attaching to the surface can be prevented by targeting cell-surface-associated adhesins (appendages, proteins and EPS). Many bacteria rely heavily on type 1 pili through FimH, curli fibers and Antigen 43 to mediate attachment on abiotic surfaces [79]. Small molecules like peptides and mannosides that have targeted these different adhesins have shown efficacy in prevention and treatment of both bacterial and fungal biofilm infections. Studies reported mannosides targeting the bacterial adhesin FimH prevented catheter-associated urinary tract infection (UTI) in mice [80]. Pharmaceutically active surface coatings have long been investigated to both prevent adhesion to an implanted device and kill planktonic bacteria in the vicinity of the device, thereby reducing the formation of biofilm. However, caution must be exercised when taking this approach due to the persistent presence of antibiotic that may lead to selection of antibiotic resistance in the absence of infection or by inappropriate use of antibiotics [81]. Conversely, the production of EPS and cellular division can be inhibited. The production of EPS involves both extracellular and intracellular signaling networks as well as non-signaling mechanisms. Cyclic-di-GMP (c-diGMP) and cyclic-di-AMP (c-di-AMP) were found to control various EPS producing exoenzymes, polysaccharides and adhesins, making these molecules potential candidates to inhibit EPS [82,83]. EPS is synthesized by a virulence factor called glucosyltransferases (Gtfs) in pathogens like Streptococcus mutans, which forms the glucan layer of the biofilm. Studies showed that using small-molecule inhibitors of glucosyltransferase, there was a decrease in the accumulation of pathogenic biofilms on teeth [84]. These small molecule inhibitors when used in combination with other approaches like low electric fields greatly improve therapeutic efficacy $[85,86]$. In addition to titanium implant associated biofilm development with a traditional EPS composition, urinary tract infections, which are usually associated with biofilm formation, produce urease to hydrolyze urea to ammonium ions causing urinary 
obstruction. The formation of these ions leads to the precipitation of magnesium and calcium phosphate crystals, which form a protective EPS-like layer for the biofilm, while also increasing the $\mathrm{pH}$ of the urine [87]. Although not a traditional targeting strategy, specifically inhibiting the enzyme urease to prevent the production of these ions is an important alternative that should be considered in these cases. Other strategies include decreasing the urine $\mathrm{pH}$ to inhibit the formation of ions and $\mathrm{pH}$ induced delivery of antibiotics during infection. Lastly, dispersion of biofilm, the final stage in a mature biofilm, can be induced via mechanical means as free-floating, planktonic bacteria are more susceptible to therapeutic interventions.

\subsection{OTHER EMERGING APPROACHES AND CARRIERS}

\subsubsection{Bacteriophage}

Bacterial resistance to antibiotics as well as their ability to resist the host immune system is interconnected. Since bacteria adapt to the immune system, they become resistant to a spectrum of antibiotics based on the host's exposure history, but this adaptation also makes them susceptible to other novel classes of antimicrobial compounds $[88,89]$. One of the most promising approaches is the use of engineered bacteriophages to combat resistance [90]. The use of a phage as a nucleic acid drug carrier allows a targeted bacterial infection. Recent advances in CRISPR gene editing allowed the creation of a phage to remove a bacterial resistance gene and eventually kill the bacteria [91]. Nevertheless, relying on natural bacteriophage mechanisms of infection may prove susceptible to bacterial resistance.

\subsubsection{Antibiotic drug discovery and development}

Although most antibiotics are either carbohydrate based molecules or carbohydrate receptor targeting molecules, new targets and new classes of antibiotics are consistently be sought out and generated. In fact new molecules that mimic critical carbohydrates with improved properties could be a promising approach [92]. Targeting the inhibition of lipopolysaccharide (LPS), specifically the synthesis of the lipid A region, in gram-negative bacteria is such an approach. A more specific strategy to target the core oligosaccharide is the inhibition of enzymes involved in the biosynthesis of 0 -antigens, specific to different bacterial strains. Another strategy that uses unique cell-surface carbohydrates, is the design of unnatural amino acids like D-peptides, L-DNA, or L-RNA as enzymatic resistant receptors. Transglycolase is one of the key enzymes involved in the polymerization of the disaccharide units of peptidoglycan. This enzyme can be used as a target for small molecule drugs as it is easily accessible due to its location on the cell surface. With the recent 
advances in functional genomics, unique RNA sequences will serve as new drug targets. Aminoglycosides, which are primarily involved in the inhibition of protein synthesis in bacteria, may serve as the primary source of small molecules for the identification of new leads to target RNA. However, with each new drug developed a novel, microenvironment triggered or targeted delivery system should be developed in conjunction.

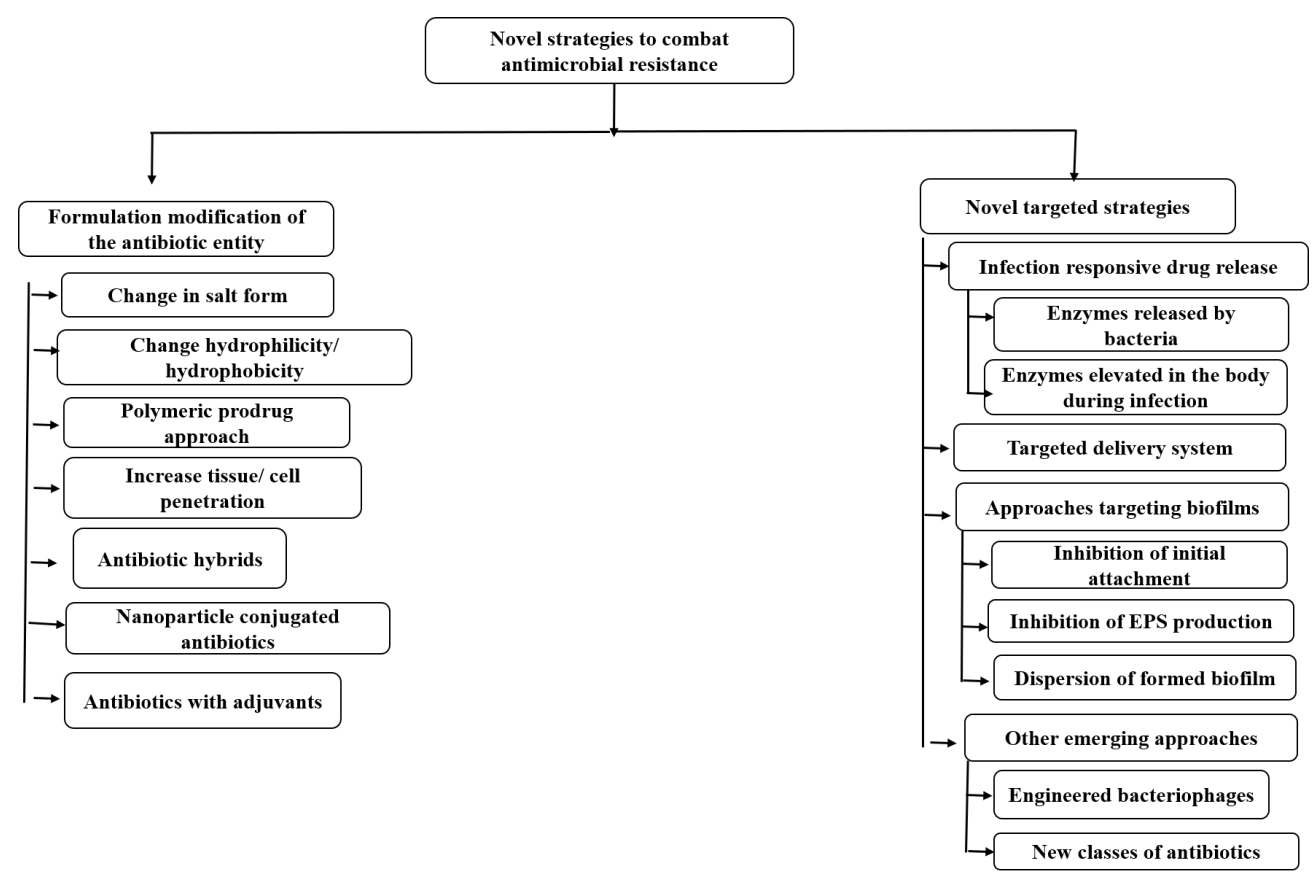

Figure 7. Over view of different strategies employed to combat antibiotic resistance

\subsection{CONCLUSION}

Although antimicrobial therapy has been one of the important advances in modern medicine, antibiotic resistance has quickly developed to become one of the greatest public health threats and identification of pipeline of new antibiotics became more challenging. Development of novel strategies along with sophisticated diagnostic technology is the need of the hour. Drug delivery strategies (Figure 7) based on a combination of infection specific mechanisms targeting represent a promising pathway forward to better utilize our current antibiotic arsenal to combat drug resistance. Although targeted delivery and formulation modifications have been successful in the research to combat resistance, combination therapies with synergistic antibiotic mechanisms and delivery strategies are continuing to gain traction. 


\section{REFERENCES}

1. C. Llor, L. Bjerrum, Ther. Adv. Drug Saf. 5(6) (2014) 229-241.

2. C.L. Ventola, Pharm. Ther. 40(4) (2015) 277-283.

3. H.H. Lee, M.N. Molla. C.R. Cantor, J.J. Collins, Nature 467(7311) (2010) 82-85.

4. Antibiotic Resistance Threats in the United States, 2013 |

Antibiotic/Antimicrobial Resistance | CDC. [Online]. Available:

https://www.cdc.gov/drugresistance/threat-report-2013/. [Accessed: 06Feb-2018].

5. M.E.A. de Kraker, A.J. Stewardson, S. Harbarth, PLOS Med. 13(11) (2016) e1002184.

6. M.S. Butler, M.A. Blaskovich, M.A. Cooper, J. Antibiot. 70(1) (2017) 3-24.

7. FDA New Molecular Entity Antibiotic Approvals, 1981-2015 - Center for Disease Dynamics, Economics \& Policy (CDDEP), Center for Disease Dynamics, Economics \& Policy (CDDEP). [Online]. Available:

https://cddep.org/tool/fda new molecular entity antibiotic approvals 1981 2015/. [Accessed: 06-Feb-2018].

8. B. Spellberg, D.N. Gilbert, Clin. Infect. Dis. Off. Publ. Infect. Dis. Soc. Am. 59 (Suppl 2) (2014) S71-S75.

9. B. Spellberg, J.G. Bartlett, D.N. Gilbert, N. Engl. J. Med. 368(4) (2013) 299-302.

10. I.M. Gould, M. Bal, Virulence 4(2) (2013) 185-191.

11. D.I. Andersson, D. Hughes, Nat. Rev. Microbiol. 8(4) (2010) 260-271.

12. J.C. Kester, S.M. Fortune, Crit. Rev. Biochem. Mol. Biol. 49(2) (2014) 91-101.

13. N. Woodford, M.J. Ellington, Clin. Microbiol. Infect. 13(1) (2007) 5-18.

14. How Evolution Explains Antibiotic-Resistant Superbugs | Columbia University Mailman School of Public Health." [Online]. Available:

https://www.mailman.columbia.edu/public-health-now/news/howevolution-explains-antibiotic-resistant-superbugs. [Accessed: 05-Feb-2018].

15. L. Fernández, R.E.W. Hancock, Clin. Microbiol. Rev. 25(4) (2012) 661-681.

16. B.D. Brooks, A.E. Brooks, Adv. Drug Deliv. Rev. 78 (2014) 14-27.

17. F.C. Tenover, Am. J. Med., 119(6, Supplement 1 S3-S10, Jun. 2006.

18. J.L. Martinez, F. Baquero, Antimicrob. Agents Chemother. 44(7) (2000) 17711777.

19. K. Poole, A.D. Russell, P.A. Lambert, Adv. Drug Deliv. Rev. 57(10) (2005) 14431445.

20. N. Høiby, T. Bjarnsholt, M. Givskov, S. Molin, O. Ciofu, Int. J. Antimicrob. Agents 35(4) (2010) 322-332.

21. P.S. Stewart, Int. J. Med. Microbiol. 292(2) (2002) 107-113.

22. S. Molin, T. Tolker-Nielsen, Curr. Opin. Biotechnol. 14(3) (2003) 255-261.

23. J.L. Balcazar, PLoS Pathog. 10(7) (2014).

24. Antibiotic Prescribing and Use in the U.S. | Antibiotic Use | CDC, 09-Apr-2018. [Online]. Available: https://www.cdc.gov/antibiotic-use/stewardshipreport/index.html. [Accessed: 06-May-2018].

25. Overuse and overprescribing of antibiotics, CIDRAP. [Online]. Available: http://www.cidrap.umn.edu/asp/overuse-overprescribing-of-antibiotics. [Accessed: 11-Feb-2018].

26. J.A. Ayukekbong, M. Ntemgwa, A.N. Atabe, Antimicrob. Resist. Infect. Control 6 (2017).

27. K.A. Brogden, Nat. Rev. Microbiol. 3(3) (2005) 238-250. 
28. IJMS | Free Full-Text | The Role of Antimicrobial Peptides in Preventing Multidrug-Resistant Bacterial Infections and Biofilm Formation | HTML. [Online]. Available: http://www.mdpi.com/1422-0067/12/9/5971/htm. [Accessed: 20-Feb-2018].

29. B. Mishra, S. Reiling, D. Zarena, G. Wang, Curr. Opin. Chem. Biol. 38 (2017) 8796.

30. M.E. Falagas, S.K. Kasiakou, L.D. Saravolatz. Clin. Infect. Dis. 40(9) (2005) 1333-1341.

31. A.O. Surov, A.N. Manin, A.P. Voronin, et al. Eur. J. Pharm. Sci. 77 (2015) 112121.

32. J.S. Reddy, S.V. Ganesh, R. Nagalapalli, et al., J. Pharm. Sci. 100(8) (2011) 31603176.

33. D.D. Lane, F.Y. Su, D.Y. Chiu, et al., Polym. Chem. 6(8) (2015) 1255-1266.

34. L. Pichavant, C. Bourget, M.-C. Durrieu, V. Héroguez, Macromolecules 44(20) (2011) 7879-7887.

35. D. Das, S. Srinivasan, A.M. Kelly, et al., Polym. Chem. 7(4) (2016) 826-837.

36. D. Das, J. Chen, S. Srinivasan, et al., Mol. Pharm. 14(6) (2017) 1988-1997.

37. V. Pokrovskaya, T. Baasov, Expert Opin. Drug Discov. 5(9) (2010) 883-902.

38. M.F. Gordeev, C. Hackbarth, M.R. Barbachyn, et al., Bioorg. Med. Chem. Lett. 13(23) (2003) 4213-4216.

39. M.M. Butler, D.L. Shinabarger, D.M. Citron, et al., Antimicrob. Agents Chemother. 56(9) (2012) 4786-4792.

40. C.J. Arnusch, A.M. Bonvin, A.M. Verel, et al., Biochemistry (Mosc.) 47(48) (2008) 12661-12663.

41. M. Mammen, S.-K. Choi, G.M. Whitesides, Angew. Chem. Int. Ed. 37(20) (1998) 2754-2794.

42. A. Mohammed Fayaz, M. Girilal, S.A. Mahdy, S.S. Somsundar, R. Venkatesan, P.T. Kalaichelvan, Process Biochem. 46(3) (2011) 636-641.

43. T. Ahmad, I.A. Wani, N. Manzoor, J. Ahmed, A.M. Asiri, Colloids Surf. B Biointerfaces 107 (2013) 227-234.

44. E. Turos, G.S. Reddy, K. Greenhalgh, et al., Bioorg. Med. Chem. Lett. 17(12) (2007) 3468-3472.

45. M.I. Konaklieva, Antibiotics 3(2) (2014) 128-142.

46. K.A. Toussaint, J.C. Gallagher, Ann. Pharmacother. 49(1) (2015) 86-98.

47. S. Shaikh, J. Fatima, S. Shakil, S.M.D. Rizvi, M.A. Kamal, Saudi J. Biol. Sci. 22(1) (2015) 90-101.

48. S.M. Drawz, R.A. Bonomo, Clin. Microbiol. Rev. 23(1) (2010) 160-201.

49. P.N.A. Harris, P.A. Tambyah, D.L. Paterson, Lancet Infect. Dis. 15(4) (2015) 475-485.

50. A.M. King, S.A. Reid-Yu, W. Wang, et al., Nature 510(7506) (2014) 503-506.

51. G.-J. A. ter Boo, D.W. Grijpma, T.F. Moriarty, R.G. Richards, D. Eglin, Biomaterials 52 (2015) 113-125.

52. E. Imbuluzqueta, E. Elizondo, C. Gamazo, et al., Acta Biomater. 7(4) (2011) 1599-1608.

53. A. Obermeier, F.D. Matl, J. Schwabe, et al., J. Mater. Sci. Mater. Med. 23(7) (2012) 1675-1683.

54. B. Holzer, U. Grüssner, B. Brückner, et al., Colorectal Dis. 5(3) (2003) 222-227.

55. J.P. Wong, H. Yang, K.L. Blasetti, G. Schnell, J. Conley, L.N. Schofield. J. Controlled Release 92(3) (2003) 265-273. 
56. J.P. Wong, G. Schnell, M. Simpson, E. Saravolac, Artif. Cells. Blood Substit. Immobil. Biotechnol. 28(5) (2000) 415-428.

57. B.F. Gilmore, J. Biotechnol. Biomater. 2(3) (2012).

58. Biotechnol. Bioeng. 113(12) 2534-2534, Dec. 2016.

59. M. Levi, T. T. Keller, E. van Gorp, H. ten Cate, Cardiovasc. Res. 60(1) (2003) 2639.

60. M. Tanihara, Y. Suzuki, Y. Nishimura, K. Suzuki, Y. Kakimaru, Y. Fukunishi, J. Pharm. Sci. 88(5) (1999) 510-514.

61. M.-H. Xiong, Y.-J. Li, Y. Bao, X.-Z. Yang, B. Hu, J. Wang. , Adv. Mater. 24(46) (2012) 6175-6180.

62. A. Hasmann, E. Wehrschuetz-Sigl, A. Marold, et al., Ann. Clin. Biochem. 50(Pt 3) (2013) 245-254.

63. D. Schiffer, G. Tegl, R. Vielnascher, et al., Biotechnol. Bioeng. 113(12) (2016) 2553-2560.

64. G.L.Y. Woo, M. Yang, H.Q Yin, F. Jaffer, M.W Mittelman, J.P Santerre, J. Biomed. Mater. Res. 59 (2002) 35-45.

65. L. Shi, H. Salamon, E.A. Eugenin, R. Pine, A. Cooper, M.L. Gennaro, Sci. Rep. 5 (2015) 18176.

66. C. Tang, L. Cui, Y. Xu, et al., Sci. Rep. 6 (2016) 37865.

67. S.D. Baines, M.H. Wilcox, Antibiotics 4(3) (2015) 267-298.

68. M. Schroeder, B.D. Brooks, A.E. Brooks, Genes 8(1) (2017) 39.

69. P. Spigaglia, Ther. Adv. Infect. Dis. 3(1) (2016) 23-42.

70. K.K. Mehta, E.E. Paskaleva, X. Wu, et al., Biotechnol. Bioeng. 113(12) (2016) 2568-2576.

71. H. Vardhan, A.R. Bhengraj, R. Jha, A. Singh Mittal, J. Biomed. Biotechnol. 2009 (2009).

72. $\quad$ S.P. Ouellette, R.A. Carabeo, Front. Microbiol. 1 (2010).

73. J. Hai, N. Serradji, L. Mouton, et al., PLOS ONE 11(2) (2016) e0150031.

74. H.T. Moghaddam, Z.E. Moghadam, G. Khademi, A. Bahreini, M. Saeidi, Int. J. Pediatrics (2016) 12.

75. M.V.S. Rajaram, M.N. Brooks, J.D. Morris, J.B. Torrelles, A.K. Azad, L.S. Schlesinger, J. Immunol. 185(2) (2010) 929-942.

76. M. Saleh, G. Longhi, P. Hanifi-Moghaddam, R. Toubassy, Mycobact. Dis. 6(1) (2016).

77. P.B. Kang, A.K. Azad, J.B. Torrelles, et al., J. Exp. Med. 202(7) (2005) 987-999.

78. M.V.S. Rajaram, E. Arnett, A.K. Azad, et al., Cell Rep. 21(1) 126-140.

79. M. Kostakioti, M. Hadjifrangiskou, S.J. Hultgren, Cold Spring Harb. Perspect. Med. 3(4) (2013) a010306.

80. H. Koo, R.N. Allan, R.P. Howlin, P. Stoodley, L. Hall-Stoodley, Nat. Rev. Microbiol. 15(12) (2017) 740-755.

81. M. Chen, Q. Yu, H. Sun, Int. J. Mol. Sci. 14(9) (2013) 18488-18501.

82. E.E. Mann, D.J. Wozniak, FEMS Microbiol. Rev. 36(4) (2012) 893-916.

83. A. Nakhamchik, C. Wilde, D.A. Rowe-Magnus, Appl. Environ. Microbiol. 74(13) 4199-4209.

84. Z. Ren, T. Cui, J. Zeng, et al., Antimicrob. Agents Chemother. 60(1) (2016) 126135.

85. S. Subramanian, K. Gerasopoulos, M. Guo, H.O. Sintim, W.E. Bentley, R. Ghodssi, Biomed. Microdevices 18(5) (2016)95. 
86. S. Subramanian, K. Gerasopoulos, H.O. Sintim, W.E. Bentley, R. Ghodssi, A bacterial biofilm combination treatment using a real-time microfluidic platform, in 2015 Transducers - $201518^{\text {th }}$ International Conference on SolidState Sensors, Actuators and Microsystems (TRANSDUCERS), 2015, pp. 22162219.

87. S.M. Soto, Adv. Biol. (2014) [Online]. Available: https://www.hindawi.com/journals/ab/2014/543974/. [Accessed: 13-Feb2018].

88. Resistance to antibiotics and to immune system are interconnected in bacteria. [Online]. Available: https://phys.org/news/2016-06-resistance-antibioticsimmune-interconnected-bacteria.html. [Accessed: 21-Nov-2017].

89. P. Durão, D. Gülereşi, J. Proença, I. Gordo, Antimicrob. Agents Chemother. 60(7) (2016) 4324-4332.

90. S. Reardon, Nat. News 546(7660) (2017) 586.

91. M. Rodrigues, K. Hullahalli, K. Palmer,. FASEB J. 31(1_supplement) (2017) 909.3-909.3.

92. T.K. Ritter, C.-H. Wong, Angew. Chem. Int. Ed. 40(19) (2001) 3508-3533.

(C)2018 by the authors; licensee IAPC, Zagreb, Croatia. This chapter is an open-access publication distributed under the terms and conditions of the Creative Commons Attribution license (http://creativecommons.org/licenses/by/3.0/) (cc)) BY 
